\title{
Electromyography Activity of Selected Leg-dominant Lower Limb Muscles during Stance Phase of Running on Treadmill and Overground
}

\author{
Amir Reza SEDIGHI ${ }^{1}$, Mehrdad ANBARIAN ${ }^{*}$, Mohammad Hossein \\ GHASEMI ${ }^{1}$ \\ 1- Faculty of Sport Sciences Sports Biomechanics Department, Bu-Ali Sina University, Hamedan, Iran. \\ 2- Faculty of Sport Sciences, Sport Biomechanics Department, Bu-Ali Sina University, Hamedan, Iran. \\ Adress Corresponding Author: Mehrdad Anbarian Sport Biomechanics Department, Faculty of Sport Sciences, Bu-Ali Sina University, Hamedan, \\ Iran.
}

\begin{abstract}
:
The aim of present study was to compare electromyography activity of selected leg-dominant lower limb muscles during stance phase of running on treadmill and overground. Fourteen male students ran at $3.3 \mathrm{~m} / \mathrm{s}$ in both treadmill and overground conditions. Electromyography activity of some selected lower limb muscle was recorded during initial $50 \%$ and terminal $50 \%$ of stance phase. Paired t-test was employed for data analyses. The results showed a significant difference in total activity of selected lower limb muscles between treadmill and overground running conditions $(\mathrm{P}<0.05)$. Rectus femoris, vastus medialis, vastus lateralis, and biceps femoris activation during overground running were found significantly higher than running on the treadmill in initial $50 \%$ stance phase $(\mathrm{P}<0.05)$. No significant electromyography change was observed for selected muscles during terminal $50 \%$ of stance phase in both treadmill and overground conditions ( $P>0.05)$. It was concluded that treadmills running condition may be possibly useful in designing specific training programs that are aimed to control or reduce lower extremity muscles activity. According to the results of this study, treadmills running condition caused lower muscle activity consequently, may increase biomechanical efficiency or used in clinical setting.
\end{abstract}

Keywords: Electromyography, lower limb muscles, running, sport surfaces, treadmill.

\section{INTRODUCTION}

Overground and treadmill running are two popular modes that used in scientific investigations, physical therapy practice and physical training raises issues on differences in running patterns on a treadmill and on overground surfaces $(1,13,12,23)$. Previous studies have been shown that different biomechanical changes are created in the user, while running on different surfaces $(9,15,14,24,21,19)$.The running's surface has been defined as the fundamental aspects in designing the exercises that should be considered $(9,24)$. Despite pattern similarity on treadmill and overground running, several studies have been shown the major differences between running on treadmill compared to overground $(5,2)$. Numerous studies have been reported that there are biomechanical and physiological differences, such as metabolic energy consumption during treadmill and overground running or walking $(6,17,26,16,20)$. For example, Nigg et al (1995) reported that most of the lower extremity kinematic variables were substantial differed depending on the individual subject's running style, running speed in treadmill and overground conditions (16). Nonetheless, Watt et al (2010) showed kinematic and kinetic patterns of walking are similar in older adults while walking on treadmill and overground situations. Conversely, while walking on the treadmill step length and stride time was shorter and joints torques was reduced compared to overground condition (26). According to the results of these sample studies, it seems that different surfaces have different effects on biomechanics of the human locomotion. 
Runners usually adapt themselves in biomechanical features such as their landing style while running on different surfaces $(24,8)$. This adaptability may associate with neuromuscular adaptation while running on different surfaces (24). However, still this is debated that what neuromuscular changes will created in result of biomechanics alterations on the treadmill (25). Hong et al. (2012) and Baur et al. (2007) reported that the maximum plantar pressure is reduced while running on the treadmill in comparison to other surfaces $(5,4)$. Though, if we accept the biomechanical differences between treadmill and overground running conditions, then we should expect changes in muscles activation. Subsequently, these changes may help to design of some specific exercise protocols with the aim of reducing the muscles activity for injured individuals (14) or increasing the level of muscles activity for increasing the exercise intensity. Although considerable studies have been devoted to kinematic and kinetic variables of the treadmill and overground running, rather less attention has been paid to muscle activations. One of the few studies carried out by Wang et al. (2014) suggests that the activity of lower limb muscles has a significant reduction while treadmill running in comparison to other surfaces (8).

In contrast, some studies have indicated that there are no differences in muscles activity between treadmill and over-ground walking (19, 2, 7). For example, Di Nardo et al (2014) evaluated the activity of lower limbs muscles while treadmill walking vs overground walking condition. They found no significant difference in the tibialis anterior muscle activity between the two conditions, but activity of gastrocnemius muscle was increased on the treadmill in footflat phase (7).

Nevertheless, the different subjects, different research methods, evaluating the different muscles in the body and substantial differences in the objectives of the researches could create these contradictory results. Furthermore, the results of related studies of the walking cannot be extended to other activities such as running. Therefore, this study aimed to compare electromyography activity of selected leg-dominant lower limb muscles during stance phase of treadmill and overground running conditions.

\section{MATERIAL \& METHOD}

Subjects: A total of 14 male students (age: $22.5 \pm 5.5$, weight: $6.7 \pm 66.6 \mathrm{~kg}$, height: $177 \pm 7 \mathrm{~cm}$, dominant leg: right) were selected to participated in this study. Subjects were free of any cardiovascular pathology, neurological disorders, lower extremity injuries, and foot or ankle surgeries. Also, all subjects had normal foot posture with no foot deformities. Participants were active recreational runners engaging in training at least three times per week whilst completing a minimum of $25 \mathrm{~km}$ per week and had previous experience of treadmill running (22). Bu Ali Sina University Graduate Studies and Research Council (The code of approval: 1184255, Date: 2015), in agreement with the Declaration of Helsinki, approved all the procedures before the beginning of the investigation. Subjects enrolled in this study after they agreed to contribute, all procedures were explained, and informed written consent was obtained.

Procedures: Surface electromyography (sEMG) signals were collected using a 16-channel electromyography system (Biomonitor ME6000 T16, Mega Electronics Ltd., Kuopio, Finland) at $2000 \mathrm{~Hz}$ sampling rate and a signal-to-noise ratio of over 110 $\mathrm{db}$. Before placement of the electrodes (disposable electrodes of $\mathrm{Ag} / \mathrm{AgCl}$ with the conductive gel), subjects' skin was prepared with shaving hair in the site and the skin was cleaned with alcohol wipe to reduce the electrical resistance of the skin (24).

The electrodes were placed on the vastus medialis (VM), rectus femoris (RF), vastus lateralis (VL), biceps femoris (BF), gastrocnemius lateralis (GL) and tibialis anterior (TA) muscles of the dominant leg of the participants according to the SENIAM recommendations (10). A ground electrode was placed on the tibial tuberosity. The electrodes placed in the interface between the nerve center of the muscle and distal tendon. Center to center spacing of the electrodes was $20 \mathrm{~mm}$ (24) (Figure 1). The reason for choosing these muscles is because of their important roles in running and also, the availability of them in surface electromyography. Subjects' dominant legs were determined by using three tests of hitting the ball, stepping up, and restoring the balance, at the beginning of their entrance to the laboratory. Foot that was used commonly (for at least 2 out of 3 tests) was identified as the dominant leg (18). 


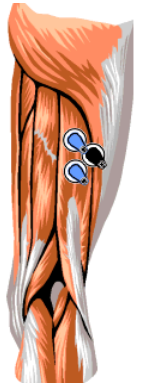

(a)

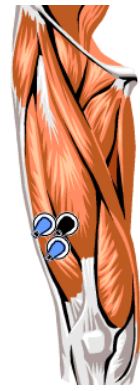

(b)

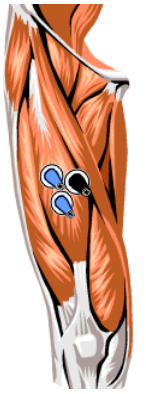

(c)

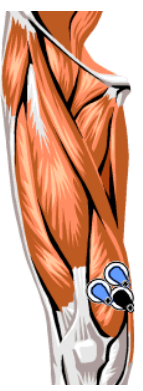

(d)

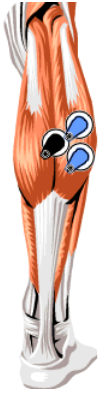

(e)

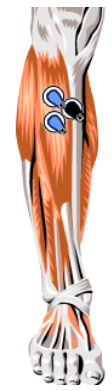

(f)

Figure 1. Electrode placement on: (a) vastus medialis, (b) rectus femoris, (c) vastus lateralis, (d) biceps femoris, (e) tibialis anterior, (f) gastrocnemius lateralis (3).

Subsequently, Maximum Voluntary Isometric Contraction (MVIC) was done to normalize the muscles activities data. Each subject asked to sit on a chair and put his knee at a flexion angle of 90 degrees, then his leg was fixed and he was asked to make every effort to do the knee extension to 5 seconds due to collect data of quadriceps MVIC. The subject was lie prone on a bed and his knee was bent to 70 degrees for hamstring; then he was asked to make every effort to do the knee extension to 5 seconds. The subject sat on the chair and he was asked to his knees and put soles of his feet against the wall for gastrocnemius (so that the angle of the foot and leg to be 90 degrees), he was asked to make every effort to do the plantar extension to 5 seconds. The subject was asked to do ankle dorsiflexion motion for 5 second in stand-up mode for tibialis anterior. The made resistance was applied by the tester in all movements. MVIC test was done twice for each muscle and the subject was given one minute rest between each test.

Two foot switches (Motion Lab Systems, Inc., USA) were attached under the most posterior part of the heel and on the first joint of foot-toe under the shoes in order to identify the key points of the running stance phase. In the present study, stance phase was introduced as the time of external area of the posterior part of the heel crash to the ground until the separation of the first joint of the foot-toe bottom from the ground.

In the treadmill running condition, participants were given a 6-minute habitation period to run on the treadmill at a speed of 3 meters per second for warm-up and to become familiarized with treadmill running $(1,24)$. Then, they ran on the treadmill at a speed of $3.3 \mathrm{~m} / \mathrm{s}$ for $2 \mathrm{~min}$ for data collection. Five successful steps of the dominant foot stance phase during the last minute were selected for data analysis. In the overground condition, subjects ran at $3.3 \pm 5 \% \mathrm{~m} / \mathrm{s}$ across a 25 meter long laboratory floor while running speed was monitored by two sets of infrared photocells. As the other condition, five successful steps of the dominant foot stance phase selected for analysis. On each running condition, participants completed five successful trials. Both treadmill and overground running were measured based on the method of Wang and Colleagues (24). All tests was done by using same running shoes were made by Asics company, in order to eliminate the interaction effect of the surface with the shoes.

All electromyography raw were processed using Megawin software. The raw EMG signals were rectified and bandpass filtered filter with bandwidth ranging from $20 \mathrm{~Hz}$ to $500 \mathrm{~Hz}$. The linear envelope was then treated using the RMS to obtain the EMG amplitude. Then, the average EMG amplitude of each muscle was calculated. The average EMG amplitude of the muscle activation was normalized by dividing by the MVIC of each muscle while running in two phases of primary $50 \%$ and final $50 \%$ of the stance phase.

Statistical analyses: The Shapiro-Wilk statistic for each condition was used to demonstrate normal distribution. Paired t-tests were utilized with an adjusted $a$ level of $\mathrm{p} \leq 0.01$ based on the number of comparisons made for each muscle at the first 50\% and the final $50 \%$ of running stance phase. All statistical procedures were conducted by SPSS 18.0 (SPSS, Inc., Chicago, IL, USA). 


\section{DISCUSSION \& CONCLUSION}

Comparing the muscles activity amount at the first $50 \%$ of the stance phase: At the first half of the stance phase of running, the muscle activation of rectus femoris, vastus lateralis, vastus medialis and biceps femoris significantly decreased in treadmill condition compared to overground running (Table 1). For tibialis anterior and gastrocnemius lateralis, treadmill condition exhibited lower muscle activation, but there was no significant.

Table 1. Mean and SD of normalized electromyography activity from selected leg-dominant lower limb muscles at the first $50 \%$ of stance phase of running on treadmill and overground.

\begin{tabular}{cccccc}
\hline Muscle & Overground & Treadmill & t-test value & p value & $\omega^{2}$ \\
\hline Rectus femoris & $39.2 \pm 17.6$ & $23.9 \pm 8.7$ & 4.26 & 0.001 & 0.38 \\
Vastus medialis & $59.3 \pm 32.8$ & $40 \pm 17.8$ & 3.26 & 0.006 & 0.256 \\
Vastus lateralis & $56.1 \pm 18.9$ & $35.1 \pm 10$ & 6.02 & 0.001 & 0.558 \\
Biceps femoris & $36 \pm 19$ & $22.3 \pm 16.9$ & 3.47 & 0.004 & 0.284 \\
Tibialis anterior & $16 \pm 7$ & $9.4 \pm 7.2$ & 1.63 & 0.126 & - \\
Gastrocnemius lateralis & $64.7 \pm 29.7$ & $52.1 \pm 20.3$ & 1.81 & 0.92 & -
\end{tabular}

Comparing the muscles activity amount at the $50 \%$ of the stance phase. No significant difference final $50 \%$ of the stance phase: As Table 2 shows, there was significant decreased activation for gastrocnemius lateralis, vastus medialis and rectus was found between treadmill and overground running conditions for other selected muscles activation femoris muscles in treadmill condition at the final

Table 2. Mean and SD of normalized electromyography activity from selected leg-dominant lower limb muscles during the final $50 \%$ of stance phase of running on treadmill and overground.

\begin{tabular}{cccccc}
\hline Muscle & Overground & Treadmill & t-test value & p value & $\omega^{2}$ \\
\hline Rectus femoris & $18.4 \pm 12.7$ & $6.9 \pm 4.1$ & 4.061 & 0.001 & 0.356 \\
Vastus medialis & $26.3 \pm 31.3$ & $20.8 \pm 10.5$ & 2.954 & 0.01 & 0.216 \\
Vastus lateralis & $28.7 \pm 30$ & $11.2 \pm 7.5$ & 2.327 & 0.037 & 0.232 \\
Biceps femoris & $31.7 \pm 17.2$ & $35.8 \pm 22$ & -1.253 & -0.391 & -702 \\
Tibialis anterior & $8.5 \pm 3.6$ & $9.4 \pm 4.2$ & 4.058 & 0.001 & 0.356 \\
Gastrocnemius lateralis & $52 \pm 20.8$ & $38.8 \pm 14.3$ & &
\end{tabular}

The aim of this study was to compare the EMG activity of the selected lower limbs muscles in stance phase of running on the treadmill and overground surfaces. The results of this study showed that there is a significant difference in the activity amount of the lower limbs muscles in the primary $50 \%$ of stance phase between two different surfaces. Activities amount of rectus femoris, vastus medialis, vastus lateralis; biceps femoris was significantly higher while running at the overground running condition than while running on the treadmill. The activities amount of gastrocnemius and tibialis anterior increased insignificantly in comparison to running position in gym on the treadmill. Also, there was no significant difference in activity amount of the lower limbs muscles in final $50 \%$ of stance phase between both surfaces. While running at the gym, activities of rectus femoris, vastus medialis, and vastus lateralis are significantly higher than while running on the treadmill, but biceps femoris and tibialis anterior activities was increased insignificantly while running on the treadmill in comparison to the overground. The activity amount of the muscles has been different in both surfaces, according to the biomechanical differences of running on the treadmill and over ground and exercising has different effects on the activity amount of the lower limbs muscles. This is because the runners coordinate themselves with kinematic features and the surface hit force while running on different surfaces $(24,8)$ and this coordination is associated with the neuromuscular changes in running on different surfaces (25). However, still this is debated that what neuromuscular changes will created in result of biomechanical changes on the treadmill (24). Results of Hong et al. (2012) and Baur et al (2007) showed that the maximum pressure of foot and the maximum force of foot plantar are reduced while running on the treadmill in comparison to other surfaces $(5,4)$. 
According to the results of their studies and the observed differences in this study, it can be concluded that while running on the treadmill reduces the ground reaction force and plantar pressure force consequently reduces muscle activity. This issue can be useful in designing the specific exercise program for injured individuals or people who require less activity in their lower limbs. Wang et al (2014), evaluated the activity of lower limbs muscles while running on the treadmill in comparison to other surfaces such as cement and natural grass. They reported that the activity of lower limbs muscles significantly reduced while running on the treadmill in comparison to other surfaces. They concluded that the kinematic adjustment of the lower extremity may explain the electromyography difference when running on different surfaces (24). Lee et al (2008) showed that the tibialis anterior and gastrocnemius activities decrease while walking on the treadmill and as a result the rehabilitation exercises can be done on the treadmill for people with nerve damages (14). Moreover, Hunter et al (2014) evaluated the activity of lower limbs muscles while running on the treadmills with the positive pressure and observed that most muscles showed decreases in activation as more body weight was supported. So these kinds of treadmills may useful intervention for certain running related injuries (12).

However, some studies also have been showed no significant difference (or increase) in the amount of muscle activity between the treadmill in comparison to other surfaces $(7,2,19,13)$. For example, Arsenault et al (1986) showed that the activities amount of soleus, rectus femoris, biceps femoris, vastus medialis and tibialis anterior increased while walking on the treadmill in comparison with walking overground (2). Furthermore, Di Nardo et al (2014) observed no significant difference in the tibialis anterior muscle activity between the treadmill and overground walking conditions, but activity of gastrocnemius was higher on the treadmill condition (7). Kalantari et al (2015) similarly showed that walking at different speeds on the treadmill increased the activation amount of gastrocnemius, biceps femoris, half tendinous, vastus medialis and vastus lateralis and medius gluteus muscles compared to the overground (13). On the one hand, these contradictions indicate the need for further research. On the other hand, there is a difference between walking and running. Due to these reasons, the results of this research can be helpful especially for rehabilitation of injured individuals.

Our findings emphasized the activation of the lower limbs muscles decreased while running on the treadmill during the stance phase among our subjects. It seems that using of the treadmill will be useful for reducing the level of lower limbs muscles activity and designing the specific exercising plans for injured individuals. 


\section{REFERENCES}

1. Arnold BJW, Weeks BK, Horan SA. An examination of treadmill running in barefoot and shod conditions in healthy men. J Sport Sci. 2018; 11: 1-8.

2. Arsenault AB, Winter DA, Marteniuk RG. Treadmill versus walkway locomotion in humans: an EMG study. Ergonomics 1986; 29(5): 665-76.

3. Back J, Son W, Lee J, Jo S, Yi J, Bikram Pandy S. EMG Analysis of muscle activity in lower limbs of snowboarders. Inter J Bio-Sci Bio-tech. 2013; 5(6):21-32.

4. Baur H, Hirschmüller A, Müller S, Gollhofer A, Mayer F. Muscular activity in treadmill and overground running. Isokin Exer Sci 2007; 15(2): 166-71.

5. Caekenberghe IV, Segers V, Aerts P, Willems P, De Clercq D. Joint kinematics and kinetics of overground accelerated running versus running on an accelerated treadmill. J R Soc Interface. 2013; 10(84):20130222.

6. Chia LC, Licari MK, Guelfi KJ, Reid SL. Investigation of treadmill and overground runnig: implications for the measurement of oxygen cost in children with developmental coordination disorder. Gait Posture. 2014;40(3): 464-70.

7. Di Nardo F, Fioretti S. EMG-based analysis of treadmill and ground walking in distal leg muscles, ,in IN XIII mediterranean conference on medical and biological engineeing and computing 2013. 2014,January, Springer international Publishing. p. 611-4.

8. Dixon SJ, Collop AC, Batt ME. Surface effects on ground reaction forces and lower extremity kinematics in running. Med Sci Sports Exer 2000; 32(11): 1919-26.

9. Garcia-Perez JA, Perez-Soriano P, Llana S, MartinezNova A, Sanchez-Zuriaga D. Effect of overground vs treadmill running on plantar pressure: Influence of fatigue. Gait Posture 2013; 38(4): 929-33.

10. Hermens HJ, Freriks B, Disselhorst-Klug C, Rau G Development of recommendations for sEMG sensor placement procedures. J Electromyogr Kinesiol 2000; 10:361-374

11. Hoffman M, Schrader J, Applegate T, Koceja D. Unilateral postural control of the funtionally dominant and nondominant extremities of healthy subjects. J Athl Train 1998; 33(4): 319-22.

12. Hunter I, Seeley MK, Hopkins JT, Carr C, Franson JJ. EMGE activity during positive-pressure treadmill running. J Electromyogr Kinesiol 2014; 24:348-52.

13. Khademi-Kalantari K, Rahimi F, Hosseini SM, Baghban AA, Jaberzadeh S. Lower limb muscular activity during walking at different speeds: Over-ground versus treadmill walking: A voluntary response evaluation. J Bodyw Mov Ther. 2017;21(3):605-611.
14. Lee SJ, Hidler J. Biomechanics of overground vs. treadmill walking in healthy individuals. J Appl Physiol 2008; 104:747-55.

15. Montgomery G, Abt G, Dobson C, Smith T, Ditroilo M. Tibial impacts and muscle activation during walking, jogging and running when performed overground, and on motorised and non-motorised treadmills. Gait Posture. 2016; 49:120-126.

16. Nigg BM, De Boer RW, Fisher V. A kinematic comparison of overground and treadmill running. Ned Sci Sports Exerc. 1995; 27(1):98-105.

17. Parvataneni K, Ploeg L, Olney SJ, Brouwer B. Kinematic, kinetic and metabolic parameters of treadmill versus overground walking in healthy older adults. Clin Biomech, 2009; 24 95-100.

18. Rabiei M, Jafarnejhad-Gro T, Binabaji H, Hosseininejad E, Anbarian M. Assessment of postural response after sudden perturbation in subjects with genu valgum. J Shahrekord Univ Med Sci 2012; 14(2): 90-100.

19. Radmehr G, Mazaheri R, Sanjari MA, Halabchi F, Mansournia MA. Comparison of activation pattern of selected trunk muscles during over ground and treadmill walking. J Mod Rehabil 2013; 6(4): 49-58.

20. Riley PO, Paolini G, Della Croce U, Paylo KW, Kerrigan DC. A kinematic and kinetic comparasion of overground and treadmill walking in healthy subjects. Gait Posture 2007; 26: 17-24.

21. Rozumalski A, Novacheck TF, Griffith CJ, Walt K, Schwartz MH. Treadmill vs. overground running gait during chilhood: A qualitative and quantitative analysis. Gait Posture 2015; 41(2): 613-8.

22. Sinclair J, Richards J, Taylor PJ, Edmundson CJ, Brooks D, Hobbs SJ. Three-dimintional kinematic comparison of treadmill and overground running. Sports Biomech 2013; 12(3): 272-282.

23. Swanson SC, Caldwell GE. An integrated biomechanical analysis of high speed incline and level treadmill running. Med Sci Sports Exerc. 2000; 32(6):1146-55.

24. Wang L, Hong Y, Li JX. Muscular activity of lower extremity muscles running on treadmill compared with different overground surfaces. Amr J Sports Sci Med 2014; 2(4): 161165.

25. Wang L, Hong Y, Li JX. Muscular Activity of Lower Extremity Overground Surfaces. Am J Sports Sci Med 2014; 2(4):161-5.

26. Watt JR, Franz JR, Jackson K, Dicharry J, Riley PO, Kerrigan DC. A three-dimensional kinematic and kinetic comparison of overground and treadmill walking in healthy elderly subjects. Clin Biomech 2010; 25: 444-9. 